\title{
Influence du sexe de l'hôte définitif dans la filariose expérimentale à Dipetalonema viteae du hamster doré Cricetus auratus
}

\author{
par F. REYNOUARD, A. BARRABES et Ch. COMBESCOT \\ Laboratoire de Parasitologie et de Médecine tropicale. U.E.R. de Médecine, \\ U.E.R. de Pharmacie, F 37000 Tours.
}

\section{Résumé.}

Les auteurs ont étudié l'influence du sexe de l'hôte sur la parasitose expérimentale à Dipetalonema viteae du Hamster doré Cricetus auratus.

Quinze jours après contrôle de la microfilarémie des hamsters parasités, ceux-ci sont sacrifiés, puis l'intensité du parasitisme évaluée en comptant les vers mâles et les vers femelles hébergés.

La microfilarémie moyenne est significativement plus élevée chez les hamsters mâles que chez les hamsters femelles. Il en est de même pour l'intensité du parasitisme : le nombre moyen de vers adultes est plus grand lorsque la parasitose évolue chez un mâle. Cependant, le sexe du hamster n'a pas d'influence sur la proportion des vers mâles et femelles hébergés et l'intensité de la microfilarémie observée chez les hamsters mâles semble seulement résulter de la présence chez ces derniers d'un plus grand nombre de vers.

\section{Summary.}

Influence of the sex of the final host on experimental Dipetalonema viteae filariosis in golden hamsters Cricetus auratus.

The authors have studied the influence of the sex of the host on experimental Dipetalonema viteae parasitosis in golden hamsters Cricetus auratus.

The parasited hamsters are sacrificed fifteen days after testing for microfilarial count, and then the extent of the infestation is measured by counting the number of male worms and female worms.

Accepté le 29 mai 1979. 
The average level of microfilarial count is significantly higher in male hamsters than in female hamsters. The same applies to the extent of parasitism : the average number of adult worms is higher when parasitosis occurs in males. However, the sex of the hamster has no influence on the proportion of male and female worms present and the degree of microfilarial count, observed in male hamsters seems to result only from the presence in the same of a greater number of worms.

L'influence du sexe, et plus particulièrement des hormones sexuelles sur l'aptitude d'un sujet à être parasité, retient depuis longtemps déjà l'attention des chercheurs, et c'est dans ce cadre que des travaux sont poursuivis au Laboratoire de Parasitologie de Tours. Ceux-ci ont permis notamment de mettre en évidence le rôle protecteur de l'œstradiol dans la bilharziose expérimentale à Schistosoma mansoni du hamster doré (4 et 6 ). Ces constatations nous ont incités à étendre nos investigations à d'autres parasitoses et la présente communication fait état des premiers résultats obtenus dans la filariose expérimentale à Dipetalonema viteae du Hamster doré Cricetus auratus. Ces recherches nous paraissent d'autant plus justifiées que dans l'espèce humaine, l'étude épidémiologique des filarioses a montré que les hommes et les femmes ne se comportent pas de la même façon vis-à-vis du parasite $(2,3$, $8,9,10,11)$.

\section{Protocole expérimental}

Un lot de 8 hamsters mâles et un lot de 8 hamsters femelles âgés de 3 mois et d'un poids moyen de $90 \mathrm{~g}$ sont mis en expérience.

\section{1) Infestation des hamsters.}

L'infestation des hamsters est réalisée à partir d'un hôte intermédiaire de la filaire: Ornithodorus tartakowskyi. Les acariens sont préalablement parasités lors d'un repas sanguin effectué sur un hamster porteur de la filaire (1). Nous choisissons un animal dont la microfilarémie est comprise entre 80 et 100 embryons pour $20 \mu \mathrm{l}$ de sang. Il s'agit du taux d'embryonnémie qui permet la meilleure infestation de l'hôte intermédiaire. En effet, si la microfilarémie est trop élevée, les ornithodores meurent et, si elle est trop faible, ils sont insuffisamment parasités pour permettre la poursuite du cycle.

Vingt-cinq jours après la contamination, les ornithodores sont dilacérés dans un liquide permettant la survie des microfilaires (Hanks Wallace ou Ringer). C'est là que nous recueillons, une à une, à l'aide d'une pipette, les larves infestantes qui sont ensuite administrées aux hamsters en expérience. Chaque animal reçoit ainsi, par voie sous-cutanée et en une seule fois, 160 larves.

(1) Cette technique a été décrite par Worms M. J., Terry R. J. et Terry A. (13). 


\section{2) Comptage des microfilaires chez les hamsters parasités.}

Soixante-quinze jours après l'inoculation des larves, la microfilarémie des hamsters est appréciée sur $20 \mu \mathrm{l}$ de sang prélevés au niveau de l'angle interne de l'œil après effondrement du sinus rétro-orbitaire. Le sang ainsi obtenu est étalé en totalité sur une lame pour préparations microscopiques, séché, hémolysé et enfin coloré au Giemsa. Le nombre total de microfilaires présentes dans $20 \mu \mathrm{l}$ de sang peut ainsi être déterminé avec précision pour chaque hamster en expérience.

\section{3) Comptage des filaires adultes chez les hamsters parasités.}

Quatre-vingt-dix jours après l'infestation, nous comptons les filaires adultes qui, après avoir terminé leur migration chez le hamster, sont essentiellement localisées dans le tissu cellulaire sous-cutané et les aponévroses. Dans ce but, les animaux sont sacrifiés et écorchés, puis une recherche des macrofilaires est effectuée dans leurs tissus après dilacération. Les vers sont prélevés à la pince et placés dans le liquide de survie ci-dessus mentionné. Dans un deuxième temps, la peau et la dépouille sont plongés, pendant une nuit, dans ce même liquide afin de permettre aux vers encore présents chez l'animal de se libérer des tissus; on les retrouvera le lendemain au fond du récipient. Les filaires adultes recueillies au cours des deux précédentes manipulations sont alors comptées et la proportion des vers de chaque sexe déterminée pour chaque animal en expérience.

Les résultats obtenus sont consignés dans les tableaux I, II, III et IV.

\section{Résultats}

Le tableau I rend compte du nombre de microfilaires trouvées dans $20 \mu \mathrm{l}$ de sang chez les 7 hamsters mâles et les 8 hamsters femelles encore en vie à ce stade de

Tableau I. - Evaluation de la microfilarémie chez les hamsters en fonction du sexe de l'hôte.

\begin{tabular}{|c|c|c|c|c|c|c|c|c|c|c|c|c|c|c|c|c|}
\hline \multirow{2}{*}{$\begin{array}{c}\text { Numéro } \\
\text { des hamsters }\end{array}$} & \multicolumn{8}{|c|}{ Hamsters $\sigma^{\prime}$} & \multicolumn{8}{|c|}{ Hamsters $Q$} \\
\hline & 1 & 2 & 3 & 4 & 5 & 6 & 7 & 8 & 9 & 10 & 11 & 12 & 13 & 14 & 15 & 16 \\
\hline $\begin{array}{c}\text { Nombre- } \\
\text { de microfilaires } \\
\text { pour } 20 \mu \text { lde sang }\end{array}$ & 33 & 60 & 58 & 42 & 179 & : & 79 & 0 & 12 & 4 & 8 & 26 & 19 & 16 & 2 & 18 \\
\hline Moyenne & \multicolumn{8}{|c|}{64,4} & \multicolumn{8}{|c|}{13,13} \\
\hline d.d.l. & \multicolumn{16}{|c|}{13} \\
\hline Test $t$ & \multicolumn{16}{|c|}{2,56} \\
\hline
\end{tabular}


l'expérience. Le nombre moyen de microfilaires pour l'ensemble des animaux constituant chacun des deux lots figure également sur ce tableau. La valeur statistique de la différence observée entre ces deux moyennes a été estimée à l'aide du test $t$ de Student.

Il ressort tout d'abord de ces résultats que le nombre moyen de microfilaires comptées dans le sang des hamsters mâles est très supérieur à celui qui est observé chez les femelles, et la différence des moyennes est significative (mieux que 0,05).

On remarquera également que chez les mâles la dispersion des résultats autour de la moyenne est importante (écart type $=56,33$ ) avec comme extrêmes 0 et 179 . Chez les hamsters femelles, les résultats sont beaucoup mieux groupés (écart type $=8,17)$ avec des extrêmes de 2 et 26 .

Tableau II. - Evaluation du nombre de filaires adultes mâles chez les hamsters en fonction du sexe du hamster.

\begin{tabular}{|c|c|c|c|c|c|c|c|c|c|c|c|c|c|c|c|c|}
\hline \multirow{2}{*}{$\begin{array}{c}\text { Numéro } \\
\text { des hamsters }\end{array}$} & \multicolumn{8}{|c|}{ Hamsters $\sigma^{\prime}$} & \multicolumn{8}{|c|}{ Hamsters $q$} \\
\hline & 1 & 2 & 3 & 4 & 5 & 6 & 7 & 8 & 9 & 10 & 11 & 12 & 13 & 14 & 15 & 16 \\
\hline $\begin{array}{l}\text { Nombre } \\
\text { de filaires } \\
\text { adultes } O^{\prime}\end{array}$ & 30 & 31 & : & 21 & 23 & $\begin{array}{l}.00 \\
.0 .0 \\
0\end{array}$ & 13 & 16 & 6 & 21 & 11 & 芯 & 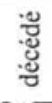 & 2 & 7 & 15 \\
\hline Moyenne & \multicolumn{8}{|c|}{22,33} & \multicolumn{8}{|c|}{10,33} \\
\hline d.d.I. & \multicolumn{16}{|c|}{10} \\
\hline Test $t$ & \multicolumn{16}{|c|}{2,94} \\
\hline
\end{tabular}

Le tableau II indique le nombre de filaires adultes (macrofilaires) de sexe mâle trouvées chez les 12 hamsters encore vivants à l'issue de l'expérience, soit 6 mâles et 6 femelles, ainsi que la moyenne des vers mâles pour l'ensemble des animaux

Tableau III. - Evaluation du nombre de filaires adultes femelles chez les hamsters en fonction du sexe du hamster.

\begin{tabular}{|c|c|c|c|c|c|c|c|c|c|c|c|c|c|c|c|c|}
\hline \multirow{2}{*}{$\begin{array}{l}\text { Numéro } \\
\text { des hamsters }\end{array}$} & \multicolumn{8}{|c|}{ Hamsters $\sigma^{\prime}$} & \multicolumn{8}{|c|}{ Hamsters $Q$} \\
\hline & 1 & 2 & 3 & 4 & 5 & 6 & 7 & 8 & 9 & 10 & 11 & 12 & 13 & 14 & 15 & 16 \\
\hline $\begin{array}{l}\text { Nombre } \\
\text { de filaires } \\
\text { adultes } Q\end{array}$ & 19 & 26 & : & 13 & 20 & ".ّूँ & 20 & 12 & 9 & 14 & 8 & : & :ृँّ & 4 & 3 & 10 \\
\hline Moyenne & \multicolumn{8}{|c|}{18} & \multicolumn{8}{|c|}{8} \\
\hline d.d.l. & \multicolumn{16}{|c|}{10} \\
\hline Test $t$ & \multicolumn{16}{|c|}{3,76} \\
\hline
\end{tabular}


constituant chacun des deux lots. Il est à noter que cette moyenne est de 22,33 pour les hamsters mâles et de 10,33 pour les hamsters femelles. La différence de ces deux moyennes est très significative (mieux que 0,01 ) ainsi qu'en témoigne l'étude statistique par le test de Student.

Le tableau III est établi de la même façon que le tableau II, mais il concerne cette fois le nombre de filaires adultes femelles qui ont été dénombrées chez les hamsters en expérience. Là encore, la différence des moyennes calculée pour l'ensemble des animaux constituant chaque lot est importante. Elle fait ressortir que si 18 vers femelles sont retrouvés en moyenne chez les hamsters mâles, le nombre correspondant pour les hamsters femelles n'est que de 8 . La différence est très significative (mieux que 0,01).

Le tableau $I V$ indique pour chaque animal en expérience la microfilarémie moyenne pour un ver femelle. Il fait également état de la moyenne de la filarémie,

Tableau IV. - Nombre de microfilaires émises par les filaires adultes femelles en fonction du sexe de l'hôte.

\begin{tabular}{|c|c|c|c|c|c|}
\hline & & $\begin{array}{l}\text { Nombre } \\
\text { de filaires } Q \\
\text { adultes }\end{array}$ & $\begin{array}{l}\text { Microfilarémie } \\
\text { pour } \\
20 \mu 1\end{array}$ & $\begin{array}{c}\begin{array}{c}\text { Microfilaremie } \\
\text { moyenne }\end{array} \\
\text { pour une filaire } ᄋ\end{array}$ & $\begin{array}{c}\text { Moyenne } \\
\text { de la microfilarémie } \\
\text { pour une filaire } Q \\
\text { calculée sur } \\
\text { l'ensemble du lot }\end{array}$ \\
\hline \multirow{9}{*}{$\begin{array}{l}\overline{-} \\
\vdots \\
\vdots\end{array}$} & Hamsters of & & & & \multirow{9}{*}{3,29} \\
\hline & $\mathrm{N}^{\circ} \mathrm{I}$ & 19 & 33 & 1,73 & \\
\hline & $\mathrm{N}^{\circ} 2$ & \multirow[t]{2}{*}{26} & 60 & \multirow[t]{2}{*}{2,69} & \\
\hline & $\mathrm{N}^{\circ} 3$ & & décédé & & \\
\hline & $\mathrm{N}^{\circ} 4$ & 13 & 42 & 3,23 & \\
\hline & No 5 & \multirow[t]{2}{*}{20} & 179 & \multirow[t]{2}{*}{8,15} & \\
\hline & $N^{\circ} 6$ & & décédé & & \\
\hline & $N^{\circ} 7$ & 20 & 79 & 3,95 & \\
\hline & $\mathrm{N}^{\circ} 8$ & 12 & 0 & 0 & \\
\hline \multirow{9}{*}{ 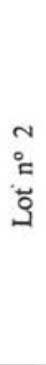 } & Hamsters $Q$ & & & & \multirow{9}{*}{1,50} \\
\hline & $\mathrm{N}^{\circ} 9$ & 9 & 12 & 1,33 & \\
\hline & $\mathrm{N}^{0} 10$ & 14 & 4 & 0,23 & \\
\hline & $N^{0} 11$ & \multirow[t]{3}{*}{8} & 8 & \multirow[t]{3}{*}{1} & \\
\hline & $N^{\circ} 12$ & & décédé & & \\
\hline & $N^{\circ} 13$ & & décédé & & \\
\hline & $\mathrm{N}^{\circ} 14$ & 4 & 16 & 4 & \\
\hline & $N^{\circ} 15$ & 3 & 2 & 0,66 & \\
\hline & $\mathrm{N}^{\circ} 16$ & 10 & 18 & 1,80 & \\
\hline & d.d.l. & 10 & & & \\
\hline & Test $t$ & 1,43 & & & \\
\hline
\end{tabular}


pour un ver femelle, mais calculé cette fois sur l'ensemble des animaux constituant le lot $n^{\circ} 1$ (hamsters mâles) et le lot $n^{\circ} 2$ (hamsters femelles). L'examen de ce tableau fait ressortir une importante dispersion des résultats autour de chacune des deux moyennes (écart type pour le lot de hamsters mâles : 2,75; écart type pour le lot de hamsters femelles : 1,34). La microfilarémie moyenne rapportée à une femelle est de 3,29 lorsque cette femelle est hébergée par un hamster mâle alors qu'elle n'est que de 1,50 lorsque le ver femelle est hébergé par un hamster femelle. Néanmoins, l'étude statistique des résultats à l'aide du test $t$ fait ressortir que ces deux moyennes ne diffèrent pas d'une façon significative (au seuil de 0,05).

Enfin, il apparaît à la lecture des tableaux II et III que si l'hôte définitif est un hamster mâle, le nombre moyen de vers mâles est de 22,33, tandis que le nombre moyen de vers femelles est de 18, différence qui n'est pas significative $(t=1,20$ et d.d.l = 10). L'examen des mêmes tableaux révèle d'autre part que si l'hôte définitif est un hamster femelle, la différence entre le nombre moyen de vers mâles, soit 10,33, et le nombre moyen de vers femelles, soit 8 , n'est pas non plus significative $(t=0,72$, d.d.1 = 10). Il est ainsi possible de conclure que, dans les conditions de l'expérience, le sexe de l'hôte n'a pas d'influence sur la proportion relative des vers mâles et des vers femelles qui est dans les deux cas de $50 \%$ ou voisin de ce chiffre.

\section{Discussion}

La technique de comptage que nous avons utilisée pour déterminer le taux de la microfilarémie de nos hamsters donne, selon Haque et coll. (7), des résultats inférieurs à ceux obtenus par la méthode de comptage directe. Il ne semble pas que cet inconvénient puisse avoir une incidence sur nos conclusions, car nous avons seulement établi une comparaison entre nos deux lots d'animaux en expérience. Par ailleurs, si nous avons choisi le $75^{\mathrm{e}}$ jour pour apprécier la microfilarémie, c'est que cette dernière passe par un maximum à ce moment ; elle décroîtra ensuite sous l'influence des anticorps, comme en témoignent les travaux de Weiss N. (12). D'autre part, notre expérience révèle que la microfilarémie est significativement moins élevée chez les hamsters femelles que chez les hamsters mâles. Ce résultat ne pourrait-il pas s'expliquer simplement par la présence chez ces derniers d'un nombre plus élevé de filaires femelles adultes? La seconde partie de notre expérience est en faveur de cette hypothèse mais elle révèle que si chez les hamsters mâles les vers femelles sont plus nombreux, les vers mâles le sont également. Il semble donc que le sexe de l'hôte ait une influence sur l'intensité de la parasitose mais, comme nous l'avons montré, cette action s'exerce d'une façon sensiblement équivalente sur les vers des deux sexes. Ces faits sont à rapprocher de ceux déjà publiés par l'un d'entre nous concernant la proportion de schistosomes mâles et femelles se développant dans la parasitose expérimentale à Schistosoma mansoni du Hamster doré Cricetus auratus (5). Nous signalerons également les travaux de Ash et coll. (1) qui, expérimentant sur deux filaires, Brugia pahangi et Brugia malayi se développant chez le Meriones unguicu- 
latus, ont abouti à des conclusions proches des nôtres. Nous mentionnerons aussi les résultats de Haque et coll. (7) qui, chez des hamsters mâles parasités par Dipetalonema vitae, n'ont pas non plus observé de différence entre le nombre de vers mâles et le nombre de vers femelles arrivant au complet développement.

Nous avons également évoqué la possibilité d'une relation entre le sexe de l'hôte et la fécondité des vers femelles, qui serait augmentée si ces derniers sont hébergés par un hamster mâle. Les résultats de la $3^{\mathrm{e}}$ partie de l'expérience sembleraient en accord avec cette hypothèse, mais les moyennes ne diffèrent pas de façon significative.

Enfin, l'étude épidémiologique des filarioses humaines montre que la microfilarémie est, en règle générale, plus faible chez les femmes avec cependant des exceptions se manifestant soit avant la puberté, soit après la ménopause (2, 3, 9 et 10$)$. Par ailleurs, dans l'onchocercose, les nodules cutanés témoignant de la présence de vers adultes dans les tissus sont également plus souvent retrouvés chez les hommes que chez les femmes (10).

\section{Conclusion}

Au cours de ce travail sur la filariose expérimentale à Dipetalonema viteae du hamster doré Cricetus auratus, nous avons étudié l'influence du sexe de l'hôte définitif sur le développement des vers adultes et l'aptitude des femelles à émettre des microfilaires.

Les résultats obtenus ont tout d'abord montré que la microfilarémie est plus importante lorsque le parasite évolue chez un hamster mâle. Cette augmentation de la microfilarémie est en rapport avec la présence chez les hamsters mâles d'un plus grand nombre de filaires adultes femelles, mais les filaires adultes mâles sont également plus nombreuses dans ces conditions.

L'augmentation du nombre de parasites mâles et femelles constatée lorsque l'hôte définitif est un hamster mâle ne s'accompagne pas d'une modification de la proportion relative de vers mâles et femelles qui semble être voisine de $50 \%$.

\section{Bibliographie}

1. Ash L. R. (1973): Chronic Brugia pahangi and Brugia malayi infections in Meriones unguiculatus. J. Parasitol., 59, 442-447.

2. Brengues J. (1975): La Filariose de Bancroft en Afrique de l'Ouest (Bancrofti filariasis in West Africa). Mém. ORSTOM, 79, 1-299.

3. Cabrera B. D., Valeza F. (1972): Filariasis in Sablayan Island, Juban, Sorsogon. Act. Med. Philipp., 8, série 2, 145-151.

4. Combescot Ch., Barrabes A., Démaret J., Reynouard F. (1971): Rôle protecteur des hormones sexuelles dans la parasitose expérimentale à Schistosoma mansoni du hamster doré femelle. C.R. du $1^{\text {er }}$ Multicolloque européen de Parasitologie, Rennes, pp. 101-104. 
5. Combescot Ch., Barrabes A., Gerhardt R. (1975) : L'œstradiol ne modifie pas la proportion de vers mâles et femelles dans la bilharziose expérimentale à Schistosoma mansoni du Hamster doré femelle. Ann. Parasit. Hum. Comp., 50, 629-633.

6. Combescot Ch., Barrabes A., Reynouard F. (1974): Effet protecteur de l'œstradiol au cours de la parasitose expérimentale à Schistosoma mansoni chez le hamster doré femelle castré. Importance de la barrière cutanée. Ann. Parasit. Hum. et Comp., 49, 165-189.

7. Haque A., Chassoux D., Ogilvie B. M., Capron A. (1978): Dipetalonema vitae infection in hamsters: enhancement and suppression of microfilaraemia. Parasitology, 76, 77-84.

8. Niel G., Vénard P., Bruhnes G., Charmot G., Gentilini M. (1976) : La filariose de Bancroft dans l'archipel des Comores (Etude immunologique). Bull. Soc. Path. Exo., 69, 84.

9. Prod'hon J. (1972) : Etude parasitologique de la filariose de Bancroft à Anjouan. Cah. ORSTOM, Entom. Méd. Parasit., 10, 263-273.

10. Ripert Ch., Riedel D., Yang R., Fouda Onana A., Zimflou I. A. (1977) : Etude épidémiologique de l'Onchocercose dans 5 villages de la Vallée de la Sanaga (Cameroun). Bull. Soc. Path. Exot., 70, 178-186.

11. Ten Eyck D. R. (1973) : Comparison of biopsy and fluorescent antibody staining techniques for the detection and study of onchocerciasis in an Ethopian population. Am. J. Epidem., 98, 283-288.

12. Weiss N. (1970): Parasitologische and immunbiologische Untersuchungen über die durch Dipetalonema viteae erzengte Nagetierfilariose. Acta tropica, 27, 219-259.

13. Worms M. J., Terry R. J., Terry A. (1961): Dipetalonema witei, filarial parasite of the jird, Meriones lybicus. I. - Maintenance in laboratory. J. Parasitol., 47, 963-970. 